\title{
Engineering two-qubit mixed states with weak measurements
}

\author{
Parveen Kumar ${ }^{\circ}$, Kyrylo Snizhko $\mathbb{C}^{\circ}$, and Yuval Gefen \\ Department of Condensed Matter Physics, Weizmann Institute of Science, Rehovot 76100, Israel
}

(Received 21 June 2020; accepted 24 September 2020; published 19 October 2020)

\begin{abstract}
It is known that protocols based on weak measurements can be used to steer quantum systems into predesignated pure states. Here we show that weak-measurement-based steering protocols can be harnessed for on-demand engineering of mixed states. In particular, through a continuous variation of the protocol parameters, one can guide a classical target state to a discorded one, and further on, toward an entangled target state.
\end{abstract}

DOI: 10.1103/PhysRevResearch.2.042014

Introduction. A generalized quantum measurement comprises two steps: (i) switching on, and later off, an interaction Hamiltonian, coupling the quantum system and the quantum detector, leading to a unitary evolution of the combined setup over a prescribed time interval; and (ii) performing a projective measurement of the decoupled detector, which leads to a probabilistic quantum jump [1-3]. The detector readout provides information about the system's state. Measurements are designated strong (projective) or weak, based on the system-detector interaction strength. The former collapses the system to one of the eigenstates of the measured observable. By contrast, generalized (also known as weak) measurements may result in a slight nudge to the system state [4-8]. No matter how weak the measurement is, it always creates an unavoidable impact on the system state through its backaction $[9,10]$. Traditionally, this measurement-induced backaction was considered an undesirable effect since the primary purpose of a measurement is to extract information about the system without perturbing it.

Following a disparate paradigm, one may employ the measurement-induced backaction on the system's state as a means to control the system's evolution, steering [11] it toward a predesignated pure target state [12-16]. A recent work (cf., Ref. [17] and references therein) analyzed a host of protocols utilizing blind measurements [18] for engineering pure target states in single- and many-body systems.

Traditionally, mixed states are not the objective of quantum steering. In practice, nonpure states may appear due to errors or noise of steering protocols. In a paradigmatic shift, we put them here as the target of steering, emphasizing the interest in and the value of mixed states. Their quantumness can be expressed through their discord [19-22]. Discorded quantum states have been proposed as resources for various quantum information tasks: achieving quantum speedup [23,24],

Published by the American Physical Society under the terms of the Creative Commons Attribution 4.0 International license. Further distribution of this work must maintain attribution to the author(s) and the published article's title, journal citation, and DOI. remote state preparation [25], and quantum purification protocols [26,27]. Engineering predesignated mixed states is, therefore, a task of interest.

The present analysis introduces a measurement-based protocol which can be used to steer a two-qubit system to an arbitrary predesignated state (pure or mixed), independently of the system's initial state (the latter is assumed unknown). We illustrate our protocol by considering a family of target states. These, depending on the protocol parameters, may be (i) nondiscorded and nonentangled ("classical"), (ii) discorded and nonentangled, or (iii) discorded and entangled, thus providing us with a smooth navigation tool from classical to fully quantum states.

The guiding principle of our protocol is as follows. Under sufficiently weak measurement, the system evolution can be described by a Lindbladian master equation. Such an equation will have at least one (and in the present case exactly one) steady state that is approached exponentially quickly. We design the protocol such that this steady state is the target state. We first recall a measurement-based protocol whose steady state is an arbitrarily chosen pure state [17]. In order to generate a mixed state, we diagonalize the density matrix of the target state and juxtapose the protocols for stabilizing each of the density matrix's pure eigenstates. In the explicit example we consider, we find that the rate of converging toward the target state does not significantly depend on its discord or degree of entanglement, so that our protocol is equally efficient independently of the target state's usefulness for quantum information purposes.

General evolution under repeated blind measurements. Consider a quantum system in state represented by the density matrix $\rho_{s}$ and a quantum detector prepared in state $\rho_{d}^{(0)}$. Before they interact, the joint system-detector state can be written as

$$
\rho(t)=\rho_{s}(t) \otimes \rho_{d}^{(0)} \text {. }
$$

In order to perform a measurement, the system interacts with the detector via an interaction Hamiltonian $H_{s-d}$; the joint system-detector state evolves as

$$
\rho(t+\tau)=U \rho(t) U^{\dagger},
$$

where $U=\exp \left(-i H_{s-d} \tau\right)$, and $\tau$ is the interaction time. Subsequently the detector state is measured projectively, 
disentangling the composite system-detector state and generating a measurement backaction on the system state. When discarding (i.e., tracing out) the measurement readouts, a procedure we denote "blind measurement," the effect of the backaction is represented through

$$
\rho_{s}(t+\tau)=\operatorname{Tr}_{d}[\rho(t+\tau)] .
$$

Following each measurement step, the detector is reset to its initial state $\rho_{d}^{(0)}$, and then the same measurement procedure is repeated. This protocol gives rise to a nontrivial evolution of the system state.

Denoting the system-detector interaction time $\tau$ and taking the continuous time limit $\tau \rightarrow d t$, one arrives at the following differential equation for the system state evolution under blind measurements

$$
\begin{aligned}
\frac{d \rho_{s}}{d t}= & \mathcal{L}\left[\rho_{s}\right]=i \operatorname{Tr}_{d}\left(\left[\rho(t), H_{s-d}\right]\right) \\
& -\frac{1}{2} \operatorname{Tr}_{d}\left(\left[H_{s-d},\left[H_{s-d}, \rho(t)\right]\right] \tau\right) .
\end{aligned}
$$

Here $\mathcal{L}$ is the Liouvillian superoperator acting on the system state, and we dropped the terms $O\left(\tau^{2}\right)$ on the right-hand side. The first term on the right-hand side of the above equation generates unitary evolution of $\rho_{s}$, while the second term represents dissipative evolution and can be cast in the form of a Lindbladian. In other words, the above equation can be written as

$$
\begin{aligned}
\frac{d \rho_{s}}{d t}= & \mathcal{L}\left[\rho_{s}\right]=i\left[\rho_{s}(t), H_{s}\right] \\
& -\frac{1}{2} \sum_{j}\left(\left\{L_{j}^{\dagger} L_{j}, \rho_{s}(t)\right\}-2 L_{j} \rho_{s}(t) L_{j}^{\dagger}\right),
\end{aligned}
$$

where $\{\cdot, \cdot\}$ represents the anticommutator, $H_{S}$ is the effective system Hamiltonian, and $L_{j}$ are the Lindblad jump operators acting on the system state. This way, the sequence of measurements influences the system state in a quasicontinuous manner and ultimately steers it to a steady state determined by the condition

$$
\frac{d \rho_{s}^{(T)}}{d t}=\mathcal{L}\left[\rho_{s}^{(T)}\right]=0 .
$$

Steering towards a pure target state. We now recall the principles of measurement-based steering protocol of Ref. [17], focusing on the two-qubit case, at the center of our analysis. The protocol facilitates stabilizing the system in an arbitrary pure target state $\left|B_{1}\right\rangle$, corresponding to $\rho_{s}^{(T)}=\left|B_{1}\right\rangle\left\langle B_{1}\right|$.

To implement the protocol, we first select three arbitrary states $\left|B_{2}\right\rangle,\left|B_{3}\right\rangle$, and $\left|B_{4}\right\rangle$, such that together with $\left|B_{1}\right\rangle$ they form an orthonormal basis in the four-dimensional Hilbert space of the two-qubit system. Three steps now follow: In the $k$ th step, $k=1,2,3$, a measurement is performed with a system-detector coupling that is designed such that the measurement backaction steers the system away from $\left|B_{k+1}\right\rangle$. This is accomplished by choosing $H_{s-d}^{k}=$ $J\left(\left|B_{1}\right\rangle\left\langle B_{k+1}\right| \otimes \sigma^{-}+\right.$H.c. $)$, where the detector is a single qubit acted upon by the Pauli matrices $\sigma^{ \pm}=\left(\sigma_{x}^{(d)} \pm i \sigma_{y}^{(d)}\right) / 2$, and $J$ is the coupling strength (for simplicity it is the same in all the three steps). Before each measurement, the detector is initialized in $\rho_{d}^{(0)}=|\uparrow\rangle\langle\uparrow|$. With the duration of each step being $\tau$, the density matrix evolution over $d t=3 \tau$ is given by

$$
\frac{d \rho_{s}}{d t}=-\frac{g}{2} \sum_{j=1}^{4}\left(\left\{L_{j}^{\dagger} L_{j}, \rho_{s}\right\}-2 L_{j} \rho_{s} L_{j}^{\dagger}\right),
$$

where $g=J^{2} \tau / 3$, and the jump operators $L_{j}=$ $\left(1-\delta_{1 j}\right)\left|B_{1}\right\rangle\left\langle B_{j}\right|$ (we introduced $L_{1}=0$ for completeness). It follows from Eq. (7) that

$$
\frac{d \rho_{s}}{d t}=0 \Leftrightarrow \rho_{s}=\rho_{s}^{(T)}=\left|B_{1}\right\rangle\left\langle B_{1}\right|,
$$

i.e., the system is steered toward the desired pure state.

It is instructive to understand the mechanism by which the above steering works. In each step, the system-detector interaction Hamiltonian $H_{s-d}^{k}$ steers the system in a twodimensional subspace spanned by $\left|B_{1}\right\rangle$ and $\left|B_{k+1}\right\rangle$ from the state $\left|B_{k+1}\right\rangle$ to $\left|B_{1}\right\rangle$ without affecting the rest of $\left|B_{i}\right\rangle$ ( $i \neq$ $k+1)$. Since $H_{s-d}^{k}$ commutes with $\left|B_{1}\right\rangle\left\langle B_{1}\right| \otimes \rho_{d}^{(0)}$, the measurement does not disturb the system if it is in state $\left|B_{1}\right\rangle$, and the detector then remains in its initial state, $|\uparrow\rangle$. This makes $\left|B_{1}\right\rangle$ not just a steady state of the evolution, but a dark state in the terminology of Ref. [28]: Once the system is in $\left|B_{1}\right\rangle$, it is not affected by the detectors. If the system is in $\left|B_{k+1}\right\rangle$, a transition to $\left|B_{1}\right\rangle$ (accompanied by the detector state flipping to $|\downarrow\rangle$ ) happens with probability $\sin ^{2} J \tau$. Likewise, with probability $\cos ^{2} J \tau$, the detector does not flip the state and the system remains in $\left|B_{k+1}\right\rangle$. Note that if the system is initially in a (coherent or incoherent) superposition of $\left|B_{1}\right\rangle$ and $\left|B_{k+1}\right\rangle$, both detector readouts affect the system state: $|\downarrow\rangle$ state of the detector implies the system has jumped from $\left|B_{k+1}\right\rangle$ to $\left|B_{1}\right\rangle$, while $|\uparrow\rangle$ implies a change of the weights of the superposition due to different probabilities of the $\uparrow$ readout depending on the system state (this is referred in the literature as a "null weak measurement" [29-32] or by a number of different names [33-35]). Averaging over the possible detector readouts and taking the limit $J \tau \ll 1$, one obtains the master equation (7).

Steering toward a mixed target state. We now focus on the key result of the paper: steering the two-qubit system to a desired mixed state. Any mixed target state $\rho_{s}^{(T)}$ has a spectral decomposition [36]

$$
\rho_{s}^{(T)}=\sum_{i=1}^{4} p_{i}\left|B_{i}\right\rangle\left\langle B_{i}\right|,
$$

where $\left|B_{i=1, \ldots, 4}\right\rangle$ form an orthonormal basis in the two-qubit Hilbert space, and $p_{i} \geqslant 0$ is the probability of the system being in the corresponding $\left|B_{i}\right\rangle$ state, so that $\sum_{i=1}^{4} p_{i}=1$. The protocol described in the previous section can be used to steer the system to $\left|B_{1}\right\rangle$. Furthermore, by exchanging the roles of $\left|B_{1}\right\rangle$ and one of $\left|B_{i \neq 1}\right\rangle$, the protocol steers the system to the corresponding $\left|B_{i}\right\rangle$. We now show that combining the four protocols, each steering the system to one of $\left|B_{i}\right\rangle$, with appropriate coupling strengths, $J \rightarrow J_{i}$, allows us to stabilize the mixed state in Eq. (9).

A schematic experimental setup for this complex protocol is presented in Fig. 1. Each part of the protocol, steering the system toward one of the $\left|B_{i}\right\rangle$ lasts $3 \tau$. Consequently, the density matrix evolution for $d t=4 \times 3 \tau=12 \tau$ is described 


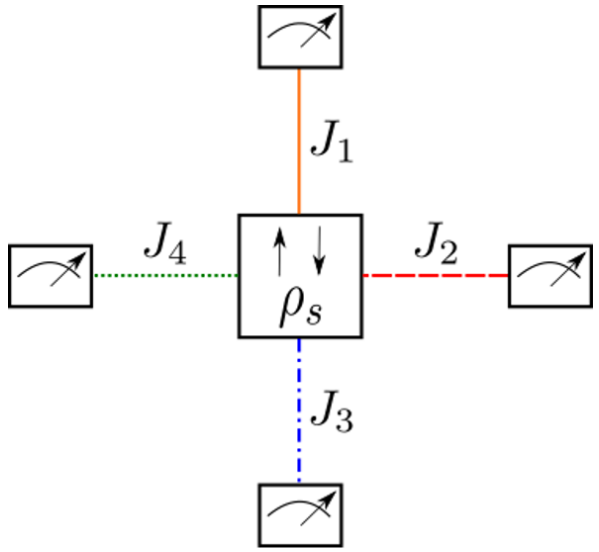

FIG. 1. Setup for steering a two-qubit system to an arbitrary mixed state. Four detectors are coupled to the system, and couplings $J_{1}, J_{2}, J_{3}$, and $J_{4}$ are switched on and off in turns such that only one of them is nonzero at any given time. Repeated measurement by any single detector would steer the system toward a pure state $\left|B_{i}\right\rangle\left\langle B_{i}\right|, i=1, \ldots, 4$. With all the detectors operating alternatingly, the system is steered toward a mixed state of the form (9).

by

$$
\begin{aligned}
\frac{d \rho_{s}}{d t} & =\mathcal{L}\left[\rho_{s}\right]=\sum_{i=1}^{4} \mathcal{L}_{i}\left[\rho_{s}\right] \\
& =-\frac{1}{2} \sum_{i=1}^{4} g_{i} \sum_{j=1}^{4}\left(\left\{L_{j}^{(i) \dagger} L_{j}^{(i)}, \rho_{s}\right\}-2 L_{j}^{(i)} \rho_{s} L_{j}^{(i) \dagger}\right),
\end{aligned}
$$

where $g_{i}=J_{i}^{2} \tau / 12$ and $L_{j}^{(i)}=\left(1-\delta_{i j}\right)\left|B_{i}\right\rangle\left\langle B_{j}\right|$. Equation (10) has a unique steady state,

$$
\rho_{s}=\rho_{s}^{(T)}=\frac{1}{\sum_{i=1}^{4} g_{i}}\left(\sum_{j=1}^{4} g_{j}\left|B_{j}\right\rangle\left\langle B_{j}\right|\right) .
$$

We note that choosing

$$
g_{j}=\bar{g} p_{j},
$$

where $j=1,2,3,4$ and $\bar{g}=\sum_{i=1}^{4} g_{i}$, stabilizes the desired target state in Eq. (9). Therefore, measurement-based steering toward an arbitrary mixed state requires diagonalizing the density matrix of the latter, bringing the state to the form (9), and concurrent utilization of the four protocols, each stabilizing one of the pure eigenstates which make up the mixed-state density matrix.

We emphasize that the simple-looking result of Eq. (12) is highly nontrivial. Indeed, for arbitrary four Lindbladians $\tilde{\mathcal{L}}_{i}$ such that $\tilde{\mathcal{L}}_{i}\left[\left|B_{i}\right\rangle\left\langle B_{i}\right|\right]=0$, one cannot guarantee that $\sum_{i} g_{i} \tilde{\mathcal{L}}_{i}\left[\sum_{j} g_{j}\left|B_{j}\right\rangle\left\langle B_{j}\right|\right]=\sum_{i, j} g_{i} g_{j} \tilde{\mathcal{L}}_{i}\left[\left|B_{j}\right\rangle\left\langle B_{j}\right|\right]=$ 0 . The fact that this holds in our case is a special feature of the measurements we employ here (i.e., of the Lindbladians describing the measurement-induced evolution). Employing other types of measurements may also allow for stabilizing arbitrary states; however, the relation between $g_{j}$ and $p_{j}$ may not be as simple as Eq. (12).

If we record the detector's readouts, the dynamics of the system subject to our protocol can be understood as follows:
The limit of weak measurement addressed here, $J_{i} \tau \ll 1$, implies that a detector click (one of the detectors flipping its state to $|\downarrow\rangle$ during the measurement) is a rare event. It follows that the system, initially prepared in a possibly coherent superposition of different $\left|B_{i}\right\rangle$, will experience coherence-preserving dynamics induced by a "no-click" backaction for a long time $\approx \bar{g}^{-1}$ (this is akin to "null weak measurements" [29-32]). Eventually, a click will register, bringing the system to one of the $\left|B_{i}\right\rangle$ states and destroying coherence between different $\left|B_{i}\right\rangle$ components. From this point on, the no-click dynamics does not affect the system state, while rare clicks make it jump between different $\left|B_{i}\right\rangle$ states. The system spending random amounts of time in different $\left|B_{i}\right\rangle$ results in the average state given by Eq. (9). Notably, the system, while being on average in the target state, probabilistically jumps among the constituent pure states $\left|B_{i}\right\rangle$, which is manifested by occasional detector clicks. In other words, a mixed target state in our protocol is a steady state but not a dark state.

Steering to "classical" versus "quantum" states. We now illustrate our protocol with an example where the system can be steered into a family of states which can be classical or quantum depending on the measurement couplings employed. Consider the following two-qubit state,

$$
\begin{aligned}
\tilde{\rho}= & p_{1}|\uparrow \uparrow\rangle\left\langle\uparrow \uparrow\left|+p_{2}\right| \psi^{+}\right\rangle\left\langle\psi^{+}\left|+p_{3}\right| \psi^{-}\right\rangle\left\langle\psi^{-}\right| \\
& +p_{4}|\downarrow \downarrow\rangle\langle\downarrow \downarrow|,
\end{aligned}
$$

where $\left|\psi^{ \pm}\right\rangle=\frac{1}{\sqrt{2}}(|\uparrow \downarrow\rangle \pm|\downarrow \uparrow\rangle)$, and

$$
\begin{aligned}
& p_{1}=\frac{[1-\beta+\alpha(1+\beta)]}{4}, \quad p_{2}=\frac{(1-\alpha)(1-\beta)}{4}, \\
& p_{3}=\frac{(1-\alpha)(1+3 \beta)}{4}, \quad p_{4}=\frac{[1-\beta+\alpha(1+\beta)]}{4} .
\end{aligned}
$$

Here $\alpha$ and $\beta$ are two independent parameters such that $0 \leqslant \alpha, \beta \leqslant 1$, and the coefficients $p_{i}$ correspond to the $p_{i}$ in Eq. (9). This state may or may not have quantum correlations depending on $\alpha$ and $\beta$. For example, using Peres-Horodecki criterion $[37,38]$, one shows that $\tilde{\rho}$ is separable (not entangled) if and only if $\alpha \geqslant \frac{3 \beta-1}{3 \beta+1}$.

Quantum correlations are commonly quantified via concurrence (a measure of entanglement) [21,39] and quantum discord [19-22]. Calculating discord $\mathcal{Q}$ and concurrence $\mathcal{C}$ for an arbitrary state is a challenging task, but for the state in Eq. (13) both of them can be calculated analytically [21,39-41]. They are respectively given by

$$
\begin{aligned}
\mathcal{Q}(\tilde{\rho})=\frac{1-\alpha}{4} & \left\{(1-\beta) \log _{2}[(1-\alpha)(1-\beta)]\right. \\
& -2(1+\beta) \log _{2}[(1-\alpha)(1+\beta)] \\
& \left.+(1+3 \beta) \log _{2}[(1-\alpha)(1+3 \beta)]\right\}
\end{aligned}
$$

and

$$
\mathcal{C}(\tilde{\rho})= \begin{cases}\frac{3 \beta(1-\alpha)-(1+\alpha)}{2} & \text { for } \alpha<\frac{3 \beta-1}{3 \beta+1}, \\ 0 & \text { otherwise. }\end{cases}
$$

Note that the discord $\mathcal{Q}(\tilde{\rho})$ is only zero when (i) $\alpha=1$ or (ii) $\beta=0$, while the concurrence $\mathcal{C}(\tilde{\rho})$ exhibits a sharp change of behavior at finite $\alpha$ and $\beta$; cf., Fig. 2. The state $\tilde{\rho}$ can thus be purely classical (both discord and concurrence vanish), discorded (concurrence vanishes), or entangled (concurrence 

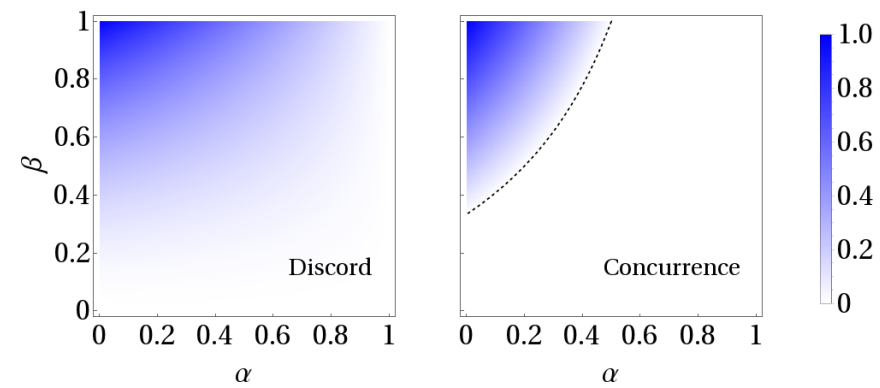

FIG. 2. Quantum discord (15) and concurrence (16) for the twoqubit state $\tilde{\rho}$ of Eq. (13) as a function of the parameters $\alpha$ and $\beta$. The black dashed line, $\alpha=\frac{3 \beta-1}{3 \beta+1}$, separates the regions of zero and nonzero concurrence. The discord is nonzero everywhere except for the lines $\alpha=1$ and $\beta=0$.

and discord are both nonzero) depending on the parameters $\alpha$ and $\beta$.

We may generate $\tilde{\rho}$ using the protocol described above. First, the target state density matrix should be diagonalized. It is evident from Eq. (13) that the eigenbasis of $\tilde{\rho}$ is

$$
\left|B_{1}\right\rangle=|\uparrow \uparrow\rangle,\left|B_{2}\right\rangle=\left|\psi^{+}\right\rangle,\left|B_{3}\right\rangle=\left|\psi^{-}\right\rangle,\left|B_{4}\right\rangle=|\downarrow \downarrow\rangle .
$$

Using Eq. (12), one obtains that the couplings $g_{i}$ in Eq. (10) are $g_{i}=\bar{g} p_{i}$, where $\bar{g}=\sum_{i} g_{i}$ characterizes the total strength of all measurements employed. Figure 3 illustrates the time evolution of the two-qubit system as it is steered from an initial state to the target state $\tilde{\rho}$. Deviations from the target state decay exponentially in time; the decay rates are determined by the real parts of the nonzero eigenvalues of the Liouvillian superoperator $\mathcal{L}$; cf., Eq. (10). The smallest of the decay rates is referred to as the convergence rate; it is determined by the inverse of the Liouvillian gap-the smallest in magnitude real part of all nonzero eigenvalues. The dependence of the convergence rate on the target state is presented in Fig. 4. Note that the dependence on $\alpha$ and $\beta$ is not too strong, implying that our protocol works equally well for both entangled and

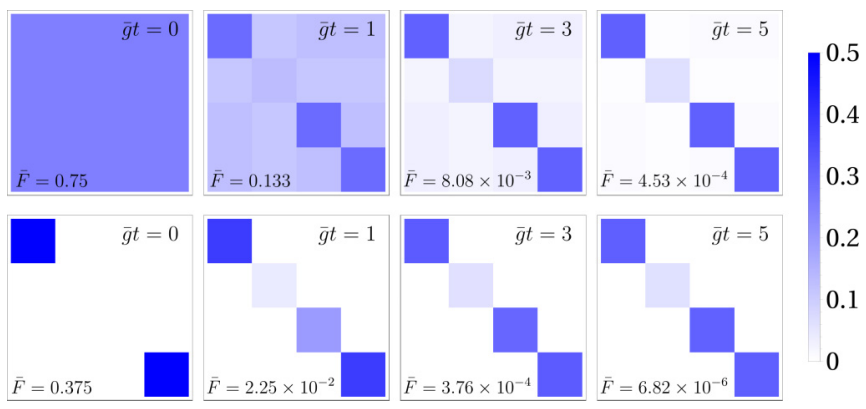

FIG. 3. The density matrix of the two-qubit system as it is steered toward a target state, $\tilde{\rho}$, of Eq. (13). The magnitudes of the density matrix entries in the basis $\left|B_{i}\right\rangle$ (17) are represented by a color scale. The initial state can be pure $\left(\rho_{s}(0)=\frac{1}{4} \sum_{i, j}\left|B_{i}\right\rangle\left\langle B_{j}\right|\right.$, top) or mixed $\left(\rho_{s}(0)=\frac{1}{2}\left(\left|B_{1}\right\rangle\left\langle B_{1}|+| B_{4}\right\rangle\left\langle B_{4}\right|\right)\right.$, bottom). $\bar{F}=1-$ $\left(\operatorname{Tr} \sqrt{\sqrt{\rho(t)} \rho^{(T)} \sqrt{\rho(t)}}\right)^{2}$ is the deviation from perfect fidelity of the target state preparation. Irrespective of the initial state, the system has essentially converged to the target state with $\alpha=\beta=\frac{1}{2}$ at $\bar{g} t=5$.

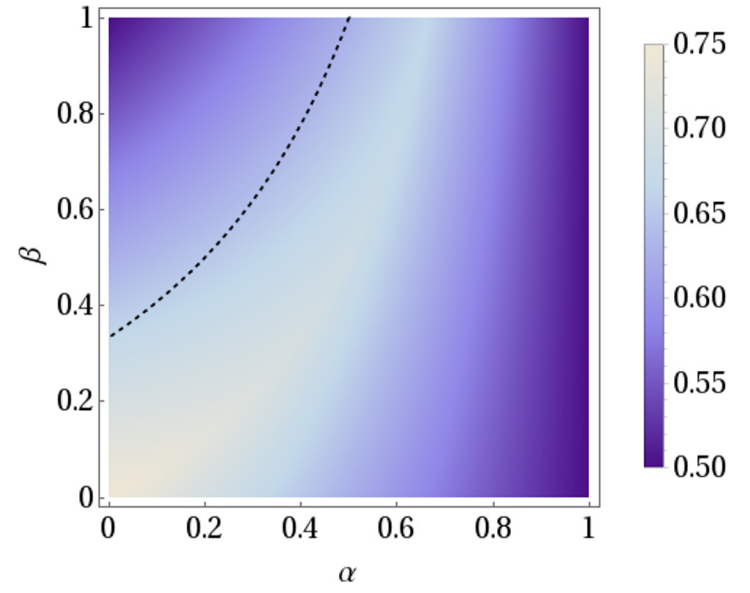

FIG. 4. The convergence rate in units of $\bar{g}$ as a function of parameters $\alpha$ and $\beta$. The black dashed line separates the regions of zero and nonzero concurrence; cf., Fig. 2 . The convergence rate does not depend significantly on the target state. In particular, it does not depend on whether the target state is classical, discorded, or entangled.

nonentangled states, as well as for states in the vicinity of the transition between the two regions.

Discussion. We have proposed a measurement-based protocol that can generate any two-qubit state by design (pure or mixed), starting from an arbitrary unknown initial state. We illustrate the protocol with an example, in which the target state can be classical, discorded, or entangled, depending on relative strengths of the measurements employed in the protocol.

Note that conventional studies of open system evolution (e.g., Refs. [42-44]) are concerned with finding the steady state of a given Liouvillian. By contrast, here we are concerned with stabilizing a predesignated state ("target state"), i.e., finding the evolution protocol (Liouvillian) for which our target state is the steady state. We also note that our approach is distinct from drive-and-dissipation schemes $[28,45-56]$, where environment is employed to relax the system to a desired state. The two crucial distinctions here are that (a) the relaxation is induced by measurements, implying the possibility to use the measurement readouts to confirm the system's desired behavior (and, possibly, hasten the convergence toward the target state), and (b) our system does not have a Hamiltonian (no "drive").

Our protocol can be implemented in a variety of experimental platforms. The main ingredient of our protocol, blind measurement stabilizing the system at a specific pure state, is particularly natural for implementation in cold-ion systems [57]. The nature of cold-ion experiments does not allow for registering individual measurement readouts in order to verify the protocol behavior. This can, however, be done by working with superconducting "artificial atoms" [58,59].

An interesting future direction concerns stabilizing $N$-qubit mixed states. Stabilizing $N$-qubit pure states in an efficient manner is already a challenging problem, which, however, has been solved for the restricted class of tensor-network states [17,46,60]. Stabilizing $N$-qubit mixed states turns out to be an even more challenging problem still under research [61]. Our 
two-qubit protocol may be a useful block in future $N$-qubit protocols.

We acknowledge funding by the Deutsche Forschungsgemeinschaft (DFG, German Research Foundation) Pro- jektnummer 277101999, TRR 183 (Project C01), and Projektnummer EG 96/13-1; by the Italia-Israel Project QUANTRA; by the Israel Science Foundation (ISF); and by the National Science Foundation-Binational Science Foundation (NSF-BSF) EAGER program.
[1] J. von Neumann, Mathematical Foundations of Quantum Mechanics, edited by N. A. Wheeler (Princeton University Press, Princeton, NJ, 2018).

[2] H. M. Wiseman and G. J. Milburn, Quantum Measurement and Control (Cambridge University Press, Cambridge, UK, 2009).

[3] J. A. Wheeler and W. H. Zurek, Quantum Theory and Measurement (Princeton University Press, Princeton, NJ, 1983).

[4] E. Arthurs and J. L. Kelly, On the simultaneous measurement of a pair of conjugate observables, Bell Syst. Tech. J. 44, 725 (1965).

[5] Y. Aharonov, D. Z. Albert, and L. Vaidman, How the Result of a Measurement of a Component of the Spin of a Spin-1/2 Particle can Turn Out to be 100, Phys. Rev. Lett. 60, 1351 (1988).

[6] T. A. Brun, A simple model of quantum trajectories, Am. J. Phys. 70, 719 (2002).

[7] B. E. Y. Svensson, Pedagogical review of quantum measurement theory with an emphasis on weak measurements, Quanta 2, 18 (2013).

[8] A. Patel and P. Kumar, Weak measurements, quantum-state collapse, and the Born rule, Phys. Rev. A 96, 022108 (2017).

[9] A. N. Korotkov, Continuous quantum measurement of a double dot, Phys. Rev. B 60, 5737 (1999).

[10] A. N. Korotkov, Selective quantum evolution of a qubit state due to continuous measurement, Phys. Rev. B 63, 115403 (2001).

[11] This is not to be confused with the term steering from the field of quantum information, which defines a special class of correlations. In this paper, we use the word in a completely different sense; steering for us is the name of the process that takes an arbitrary initial state and leads it to a predesignated target state.

[12] A. Pechen, N. Il'in, F. Shuang, and H. Rabitz, Quantum control by von Neumann measurements, Phys. Rev. A 74, 052102 (2006).

[13] L. Roa, A. Delgado, M. L. Ladrón de Guevara, and A. B. Klimov, Measurement-driven quantum evolution, Phys. Rev. A 73, 012322 (2006).

[14] L. Roa, M. L. L. de Guevara, A. Delgado, G. Olivares-Rentería, and A. B. Klimov, Quantum evolution by discrete measurements, J. Phys.: Conf. Ser. 84, 012017 (2007).

[15] K. Jacobs, Feedback control using only quantum back-action, New J. Phys. 12, 043005 (2010)

[16] S. Ashhab and F. Nori, Control-free control: Manipulating a quantum system using only a limited set of measurements, Phys. Rev. A 82, 062103 (2010).

[17] S. Roy, J. T. Chalker, I. V. Gornyi, and Y. Gefen, Measurementinduced steering of quantum systems, Phys. Rev. Res. 2, 033347 (2020).

[18] This term refers to a measurement where the final state of the detector (the readout) is traced out, discarding the acquirement of information through the measurement process but accounting for measurement backaction.
[19] L. Henderson and V. Vedral, Classical, quantum, and total correlations, J. Phys. A: Math. Gen. 34, 6899 (2001).

[20] H. Ollivier and W. H. Zurek, Quantum Discord: A Measure of the Quantumness of Correlations, Phys. Rev. Lett. 88, 017901 (2001).

[21] K. Modi, A. Brodutch, H. Cable, T. Paterek, and V. Vedral, The classical-quantum boundary for correlations: Discord and related measures, Rev. Mod. Phys. 84, 1655 (2012).

[22] M. A. Hunt, I. V. Lerner, I. V. Yurkevich, and Y. Gefen, How to observe and quantify quantum-discord states via correlations, Phys. Rev. A 100, 022321 (2019); P. Kumar and K. Snizhko, Comment on How to observe and quantify quantum-discord states via correlations, ibid. 102, 016401 (2020); M. A. Hunt, I. V. Lerner, I. V. Yurkevich, and Y. Gefen, Reply to "Comment on 'How to observe and quantify quantum-discord states via correlations",, ibid. 102, 016402 (2020).

[23] A. Datta, A. Shaji, and C. M. Caves, Quantum Discord and the Power of One Qubit, Phys. Rev. Lett. 100, 050502 (2008).

[24] B. P. Lanyon, M. Barbieri, M. P. Almeida, and A. G. White, Experimental Quantum Computing without Entanglement, Phys. Rev. Lett. 101, 200501 (2008)

[25] B. Dakić, Y. O. Lipp, X. Ma, M. Ringbauer, S. Kropatschek, S. Barz, T. Paterek, V. Vedral, A. Zeilinger, Č. Brukner, and P. Walther, Quantum discord as resource for remote state preparation, Nat. Phys. 8, 666 (2012).

[26] C. H. Bennett, D. P. DiVincenzo, J. A. Smolin, and W. K. Wootters, Mixed-state entanglement and quantum error correction, Phys. Rev. A 54, 3824 (1996).

[27] M. Murao, M. B. Plenio, S. Popescu, V. Vedral, and P. L. Knight, Multiparticle entanglement purification protocols, Phys. Rev. A 57, R4075(R) (1998).

[28] S. Diehl, A. Micheli, A. Kantian, B. Kraus, H. P. Büchler, and P. Zoller, Quantum states and phases in driven open quantum systems with cold atoms, Nat. Phys. 4, 878 (2008).

[29] R. Ruskov, A. Mizel, and A. N. Korotkov, Crossover of phase qubit dynamics in the presence of a negative-result weak measurement, Phys. Rev. B 75, 220501(R) (2007).

[30] O. Zilberberg, A. Romito, D. J. Starling, G. A. Howland, C. J. Broadbent, J. C. Howell, and Y. Gefen, Null Values and Quantum State Discrìmination, Phys. Rev. Lett. 110, 170405 (2013).

[31] O. Zilberberg, A. Romito, and Y. Gefen, Standard and null weak values, in Quantum Theory: A Two-Time Success Story (Springer, Milan, 2014), Chap. 25, p. 377.

[32] V. Gebhart, K. Snizhko, T. Wellens, A. Buchleitner, A. Romito, and Y. Gefen, Topological transition in measurement-induced geometric phases, Proc. Natl. Acad. Sci. USA 117, 5706 (2020).

[33] A. C. Elitzur and S. Dolev, Nonlocal effects of partial measurements and quantum erasure, Phys. Rev. A 63, 062109 (2001).

[34] G. S. Paraoanu, Interaction-Free Measurements with Superconducting Qubits, Phys. Rev. Lett. 97, 180406 (2006).

[35] X.-Y. Xu, J.-S. Xu, C.-F. Li, Y. Zou, and G.-C. Guo, Experimental demonstration of nonlocal effects in the partial-collapse 
measurement and reversal process, Phys. Rev. A 83, 010101(R) (2011).

[36] M. A. Nielsen and I. L. Chuang, Quantum Computation and Quantum Information (Cambridge University Press, Cambridge, UK, 2010).

[37] A. Peres, Separability Criterion for Density Matrices, Phys. Rev. Lett. 77, 1413 (1996).

[38] M. Horodecki, P. Horodecki, and R. Horodecki, Separability of mixed states: Necessary and sufficient conditions, Phys. Lett. A 223, 1 (1996).

[39] W. K. Wootters, Entanglement of Formation of an Arbitrary State of Two Qubits, Phys. Rev. Lett. 80, 2245 (1998).

[40] S. Luo, Quantum discord for two-qubit systems, Phys. Rev. A 77, 042303 (2008).

[41] M. Ali, A. R. P. Rau, and G. Alber, Quantum discord for twoqubit X states, Phys. Rev. A 81, 042105 (2010).

[42] S. Gao, Dissipative Quantum Dynamics with a Lindblad Functional, Phys. Rev. Lett. 79, 3101 (1997).

[43] T. Prosen, Exact Nonequilibrium Steady State of a Strongly Driven Open XXZ Chain, Phys. Rev. Lett. 107, 137201 (2011).

[44] V. V. Albert and L. Jiang, Symmetries and conserved quantities in Lindblad master equations, Phys. Rev. A 89, 022118 (2014).

[45] R. Wu, A. Pechen, C. Brif, and H. Rabitz, Controllability of open quantum systems with Kraus-map dynamics, J. Phys. A: Math. Theor. 40, 5681 (2007).

[46] B. Kraus, H. P. Büchler, S. Diehl, A. Kantian, A. Micheli, and P. Zoller, Preparation of entangled states by quantum Markov processes, Phys. Rev. A 78, 042307 (2008).

[47] M. Roncaglia, M. Rizzi, and J. I. Cirac, Pfaffian State Generation by Strong Three-Body Dissipation, Phys. Rev. Lett. 104, 096803 (2010).

[48] A. Pechen, Engineering arbitrary pure and mixed quantum states, Phys. Rev. A 84, 042106 (2011).

[49] S. Diehl, E. Rico, M. A. Baranov, and P. Zoller, Topology by dissipation in atomic quantum wires, Nat. Phys. 7, 971 (2011).

[50] K. W. Murch, U. Vool, D. Zhou, S. J. Weber, S. M. Girvin, and I. Siddiqi, Cavity-Assisted Quantum Bath Engineering, Phys. Rev. Lett. 109, 183602 (2012).
[51] C.-E. Bardyn, M. A. Baranov, C. V. Kraus, E. Rico, A. İmamoğlu, P. Zoller, and S. Diehl, Topology by dissipation, New J. Phys. 15, 085001 (2013).

[52] Z. Leghtas, U. Vool, S. Shankar, M. Hatridge, S. M. Girvin, M. H. Devoret, and M. Mirrahimi, Stabilizing a Bell state of two superconducting qubits by dissipation engineering, Phys. Rev. A 88, 023849 (2013).

[53] Y. Liu, S. Shankar, N. Ofek, M. Hatridge, A. Narla, K. M. Sliwa, L. Frunzio, R. J. Schoelkopf, and M. H. Devoret, Comparing and Combining Measurement-Based and DrivenDissipative Entanglement Stabilization, Phys. Rev. X 6, 011022 (2016).

[54] N. Goldman, J. C. Budich, and P. Zoller, Topological quantum matter with ultracold gases in optical lattices, Nat. Phys. 12, 639 (2016).

[55] Y. Lu, S. Chakram, N. Leung, N. Earnest, R. K. Naik, Z. Huang, P. Groszkowski, E. Kapit, J. Koch, and D. I. Schuster, Universal Stabilization of a Parametrically Coupled Qubit, Phys. Rev. Lett. 119, 150502 (2017).

[56] Z. Huang, Y. Lu, E. Kapit, D. I. Schuster, and J. Koch, Universal stabilization of single-qubit states using a tunable coupler, Phys. Rev. A 97, 062345 (2018).

[57] E. Ben Av, Y. Shapira, N. Akerman, and R. Ozeri, Direct reconstruction of the quantum-master-equation dynamics of a trapped-ion qubit, Phys. Rev. A 101, 062305 (2020).

[58] R. Vijay, D. H. Slichter, and I. Siddiqi, Observation of Quantum Jumps in a Superconducting Artificial Atom, Phys. Rev. Lett. 106, 110502 (2011).

[59] Z. K. Minev, S. O. Mundhada, S. Shankar, P. Reinhold, R. Gutiérrez-Jáuregui, R. J. Schoelkopf, M. Mirrahimi, H. J. Carmichael, and M. H. Devoret, To catch and reverse a quantum jump mid-flight, Nature (London) 570, 200 (2019).

[60] F. Verstraete, M. M. Wolf, and J. Ignacio Cirac, Quantum computation and quantum-state engineering driven by dissipation, Nat. Phys. 5, 633 (2009).

[61] D. Bondarenko and T. J. Osborne, Constructing local frustration free Lindbladians with given MPDOs as stable space (unpublished). 